\title{
Albuminuria as a risk factor for mild cognitive impairment and dementia-what is the evidence?
}

Boris Bikbov (D) ${ }^{1}$, Maria José Soler (D) ${ }^{2}$, Vesna Pešić ${ }^{3}$, Giovambattista Capasso ${ }^{4,5}$, Robert Unwin (D) ${ }^{6}$, Matthias Endres ${ }^{7}$, Giuseppe Remuzzi ${ }^{1}$, Norberto Perico ${ }^{1}$, Ron Gansevoort ${ }^{8}$, Francesco Mattace-Raso ${ }^{9}$, Annette Bruchfeld $\mathbb{D}^{10}$, Andreja Figurek ${ }^{11}$ and Gaye Hafez $\mathbb{D}^{12}$; the CONNECT Action (Cognitive Decline in Nephro-Neurology European Cooperative Target)

${ }^{1}$ Istituto di Ricerche Farmacologiche Mario Negri IRCCS, Bergamo, Italy, ${ }^{2}$ Division of Nephrology Autonomous University of Barcelona, Hospital Universitari Vall d'Hebron, Barcelona, Spain, ${ }^{3}$ Faculty of Pharmacy, University of Belgrade, Belgrade, Serbia, ${ }^{4}$ Department Translational Medical Sciences, Univ. Campania “L. Vanvitelli”, Naples, Italy, ${ }^{5}$ BIOGEM, Insititute Molecular Biology and Genetics, Ariano Irpino, Italy, ${ }^{6}$ Department of Renal Medicine, University College London, London, UK, ${ }^{7}$ Klinik und Hochschulambulanz für Neurologie, Charité-Universitätsmedizin Berlin, Berlin, Germany, ${ }^{8}$ Department of Internal Medicine, University Medical Center Groningen, Groningen, The Netherlands, ${ }^{9}$ Department of Internal Medicine, Erasmus MC University Medical Center, Rotterdam, The Netherlands, ${ }^{10}$ Unit of Renal Medicine, Linköping and Karolinska University Hospital, Stockholm, Sweden, ${ }^{11}$ Institute of Anatomy, University of Zurich, Zurich, Switzerland and ${ }^{12}$ Department of Pharmacology, Faculty of Pharmacy, Altinbas University, Istanbul, Turkey,

Correspondence to: Boris Bikbov; E-mail: boris@bikbov.ru

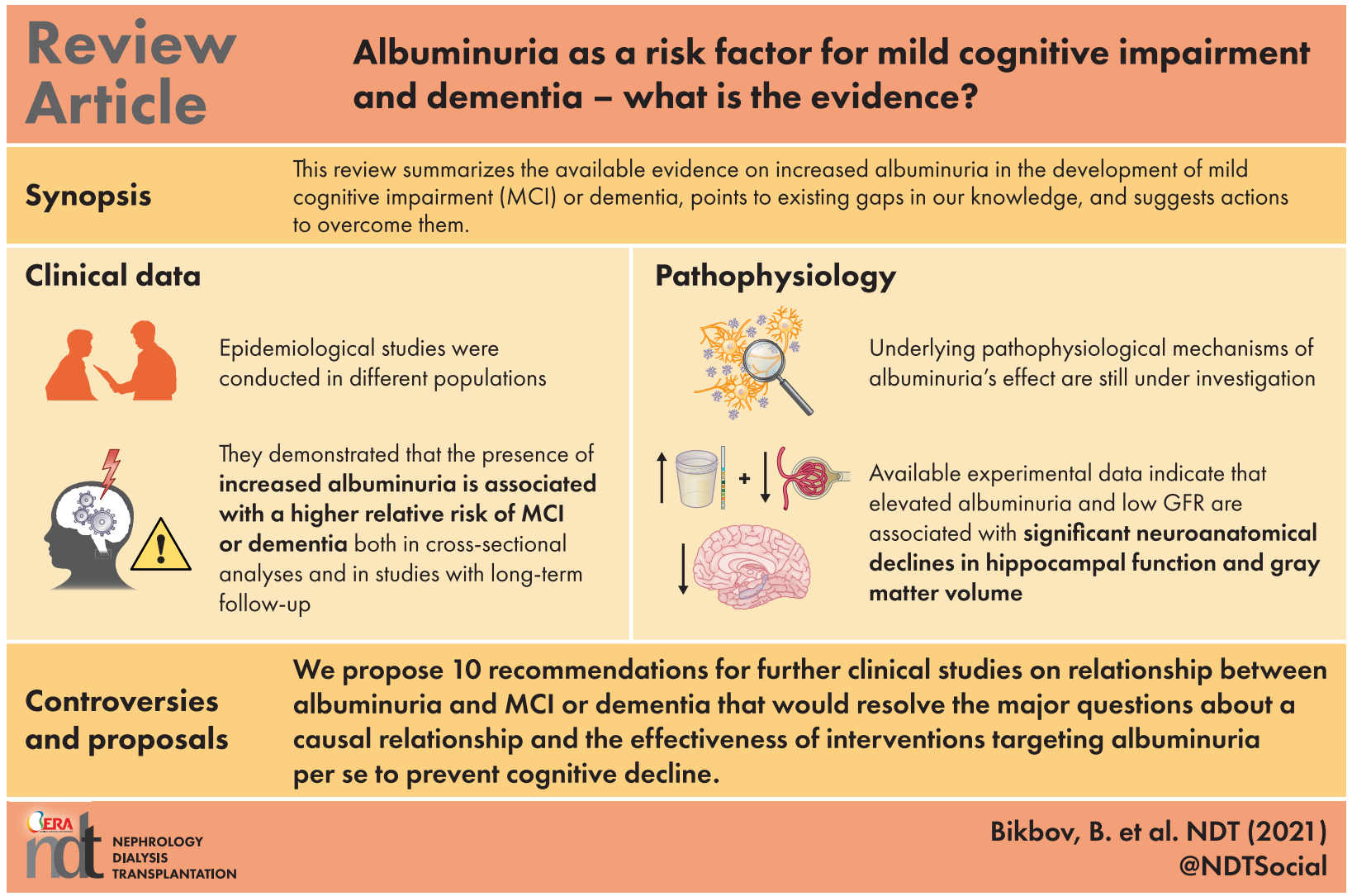




\section{ABSTRACT}

Kidney dysfunction can profoundly influence many organ systems, and recent evidence suggests a potential role for increased albuminuria in the development of mild cognitive impairment (MCI) or dementia. Epidemiological studies conducted in different populations have demonstrated that the presence of increased albuminuria is associated with a higher relative risk of MCI or dementia both in cross-sectional analyses and in studies with long-term follow-up. The underlying pathophysiological mechanisms of albuminuria's effect are as yet insufficiently studied, with several important knowledge gaps still present in a complex relationship with other MCI and dementia risk factors. Both the kidney and the brain have microvascular similarities that make them sensitive to endothelial dysfunction involving different mechanisms, including oxidative stress and inflammation. The exact substrate of MCI and dementia is still under investigation, however available experimental data indicate that elevated albuminuria and low glomerular filtration rate are associated with significant neuroanatomical declines in hippocampal function and grey matter volume. Thus, albuminuria may be critical in the development of cognitive impairment and its progression to dementia. In this review, we summarize the available evidence on albuminuria's link to MCI and dementia, point to existing gaps in our knowledge and suggest actions to overcome them. The major question of whether interventions that target increased albuminuria could prevent cognitive decline remains unanswered. Our recommendations for future research are aimed at helping to plan clinical trials and to solve the complex conundrum outlined in this review, with the ultimate goal of improving the lives of patients with chronic kidney disease.

Keywords: albuminuria, chronic kidney disease, dementia, glomerular filtration rate, mild cognitive impairment

\section{INTRODUCTION}

Kidney dysfunction can have a profound effect on many organ systems, and chronic kidney disease $(\mathrm{CKD})$ is recognized as a major risk factor for not only broader cardiovascular diseases [1], but also for pre-eclampsia, premature delivery and low birth weight [2]. Recently, evidence has emerged for a relationship between CKD and mild cognitive impairment (MCI) or dementia, and this review aims to summarize different aspects of this still poorly understood association. The magnitude of the problem is large, with almost 700 million persons worldwide affected by CKD [3] and almost 51 million with Alzheimer's disease or other dementias [4]. The burden of both CKD, MCI and or dementia increases with age, but apart from an aging population, there are additional factors that drive both conditions and lead to an increase in their age-standardized prevalence rates (which accounts for both population growth and aging) over the past 30 years: CKD and dementia have shown an increase of $9.4 \%$ and $5.7 \%$, respectively, whereas cardiovascular disease, cancer and chronic respiratory diseases decreased by $4.4 \%, 6.6 \%$ and $16.9 \%$, respectively [4].
The interest in studying the interplay between CKD and MCI or dementia has been determined by its potential for practical application, with the main question being whether the treatment of $\mathrm{CKD}$ can prevent $\mathrm{MCI}$ and dementia. Unfortunately, so far the answer is unknown, and more research and clinical studies will be needed to resolve it. Previous studies have suggested that albuminuria is related to MCI and dementia, and in this review we summarize the available evidence, point out existing gaps in our knowledge and make some suggestions on what might be done to fill them.

\section{ASSOCIATION OF INCREASED ALBUMINURIA WITH MCI AND DEMENTIA}

The first published clinical studies demonstrating an association between albuminuria and dementia were confined mainly to elderly populations [5-9]. In one early investigation in older adults from the Cardiovascular Health Cognition Study $(n=2316)$, Barzilay et al. found a cross-sectional association between increasing albuminuria and dementia. This association remained significant after adjustment for factors associated with dementia, such as hypertension, diabetes and prevalent cardiovascular disease. The authors suggested that the association of albuminuria and dementia may be partly explained by the many anatomical microvascular similarities found in the brains of people with dementia and in the kidneys of patients with albuminuria [6]. The Prevention of Renal and Vascular End-Stage Disease study conducted in a general population ( $n=4095)$ found that elevated albuminuria, but not estimated glomerular filtration rate (eGFR), was associated with worse cognitive function. However, this association was only present in the younger cohort (lower tertile of age: $42 \pm 4$ years old). Furthermore, younger subjects with an increase in albuminuria during the 6 years before cognitive function testing performed significantly worse on testing than those with stable albuminuria. The authors suggested that the stronger association of albuminuria with cognitive function in younger versus older participants is plausible, because at a young age the prevalence of interacting comorbid conditions that might confound this association is low [7]. Similarly, a high urinary albumin-tocreatinine ratio (UACR) was significantly associated with the presence of imaging markers of cerebral small vessel disease in middle-aged and elderly participants of the general populationbased Rotterdam Study [10], and with an increase of the white matter hyperintensities (reflecting deteriorating cerebral white matter due to myelin breakdown) volume to the intracranial volume ratio in the Hisayama Study [11]. More recently, this association has been confirmed in the population-based AGESReykjavik Study (mean age 75 years), showing that participants with incident albuminuria (UACR $>30 \mathrm{mg} / \mathrm{g}$ ) had $21 \%$ more white matter hyperintensity volume progression compared with participants without incident albuminuria [12]. Recent studies, including a prospective Atherosclerosis Risk in Communities (ARIC) study [13], the Hisayama Study [14] and others, also found that increased albuminuria is consistently associated with the incidence of dementia.

In diabetic patients, albuminuria has been linked to accelerated cognitive decline. Microvascular cerebral disease manifests 
as impaired vasoreactivity, hypoperfusion and decreased metabolism, which may lead to hypoxia and brain tissue loss. In middle-aged adults with diabetes and preserved baseline eGFR $\left(\backsim 90 \mathrm{~mL} / \mathrm{min} / 1.73 \mathrm{~m}^{2}\right)$, cognitive function assessed by the Digit Symbol Substitution Test was worse in participants with persistent and progressive albuminuria compared with participants with no albuminuria. Moreover, the eGFR decline was greater in those with persistent albuminuria compared with those with increasing albuminuria, suggesting a dose effect. These findings were independent of both baseline eGFR and eGFR decline [15]. Mehta et al. used 3D magnetization prepared rapid acquisition with gradient echo magnetic resonance imaging (MRI) at 3T to evaluate the effect of subclinical levels of albuminuria on grey matter (GM) in type 2 diabetes mellitus (DM). These authors found a link between UACR and GM volume changes in type $2 \mathrm{DM}$ subjects compared with agematched controls. Of note, even subclinical levels of $\mathrm{UACR} \geq 5 \mathrm{mg} / \mathrm{g}$ were associated with an early decline in brain health. This study also found that the lesions at the GM level begin early when UACR is within accepted normal limits, but there is clearly a continuum [16]. In contrast, in the Memory in Diabetes substudy of the Action to Control Cardiovascular Risk in Diabetes study of middle-aged and older adults with type 2 $\mathrm{DM}$, albuminuria at baseline and its persistence during followup were not independently associated with an increase in abnormal white matter hyperintensity volume when analyses were adjusted for systolic blood pressure (SBP) [17]. The decline in cognitive function in association with albuminuria appeared to be mediated by other risk factors such as elevated SBP, older age, oxidative stress, obesity and increased arterial stiffness. Later studies by Freedman et al. demonstrated that in African-Americans, type 2 DM, mildly elevated UACR and slightly reduced eGFR were associated with poorer mental processing speed and working memory [18]. These results identified a subgroup of African-Americans with type $2 \mathrm{DM}$ at higher risk for developing reduced cognitive function, and suggested possible treatment pathways for reducing the burden of cognitive impairment-related disability. In patients with early diabetes from the Glycaemia Reduction Approaches in Diabetes study $(n=4998)$, participants with albuminuria or eGFR $<60 \mathrm{~mL} /$ $\mathrm{min} / 1.73 \mathrm{~m}^{2}$ had significantly lower test scores for information processing speed and perception, executive function and ability to categorize information, and for verbal learning and memory compared with participants without renal disease. These findings remained statistically significant after adjustment for hypertension, dyslipidaemia and waist circumference [19].

The association of microalbuminuria and cognitive function has also been investigated in patients with human immunodeficiency virus (HIV). Patients with HIV without severe DM and/ or hypertension, hepatitis $\mathrm{C}$ virus infection, past or ongoing neurological diseases (notably acquired immunodeficiency syndrome defining neurological events) and/or alcohol or illicit drug addiction were selected for the study. After adjustment for factors associated with $\mathrm{HIV}$-associated neurocognitive disorders and/or microalbuminuria (i.e. age, educational level, hypertension and $\mathrm{CD} 4+\mathrm{T}$-cell nadir), patients with previous microalbuminuria had a worse cognitive performance for the information processing speed domain. HIV patients treated with combination antiretroviral therapy and a history of microalbuminuria had worse cognitive performance for the information processing speed domain, possibly because of the presence of cerebral small vessel disease [20].

Of particular interest is the analysis of cognitive function in the nephrotic syndrome (NS) characterized by heavy proteinuria $>3.5 \mathrm{~g} / 24 \mathrm{~h}$ (accompanied by oedema, hypercholesterolaemia, hypoalbuminaemia and prothrombotic state) and usually a normal eGFR that allows separation of the effects of proteinuria and reduced eGFR. Unfortunately, literature data are scant. In children with NS, different studies have demonstrated an altered quality of life [21], behavioural abnormalities (mostly hyperkinesis) [22], 'thought problems' and 'internalizing problems', without mood changes or attention problems [23]. However, the interpretation of these findings is confounded by concurrent factors. Thus, psychological stress may trigger proteinuria in children with steroid-sensitive NS [24] and mood changes could be associated with corticosteroid therapy for NS [23]. In adults with NS, a study conducted in Taiwan suggested a correlation between NS and ischaemic stroke [25].

\section{ROLE OF LOW EGFR IN COGNITIVE IMPAIRMENT AND DEMENTIA}

There are conflicting data on the role of eGFR in the impairment of separate cognitive domains [26]. This also concerns the evidence correlating low eGFR with overt dementia. Thus, in a retrospective community-based cohort study on geriatric patients, increased albuminuria and low eGFR have been suggested as risk factors for dementia [27]. However, the largescale population Helseundersøkelsen i Nord-Trøndelag study did not reveal a significant association between isolated low eGFR and dementia [28]. Moreover, the Rotterdam study [10] and the AGES-Reykjavik study [12] have documented, respectively, that low eGFR is independently associated with lower cerebral blood flow, and that a faster eGFR decline $(>3 \mathrm{~mL} / \mathrm{min} /$ $1.73 \mathrm{~m}^{2} /$ year) is associated with increased risk for developing manifestations of cerebral small vessel disease.

These controversial results could be partially explained by the different methods used for GFR evaluation. Of note, the widely accepted eGFR equations are based on serum creatinine level that can be affected by non-renal factors, including decreased muscle mass in elderly patients. To overcome this limitation, the ARIC study estimated GFR by three methods and found that only low eGFR calculated by equations based on cystatin $C$ and $\beta_{2}$-microglobulin was associated with higher dementia incidence, but not low eGFR based on serum creatinine [13]. This study also demonstrated the cumulative hazard in participants who had both increased UACR $(>30 \mathrm{mg} / \mathrm{g})$ and low cystatin C-based eGFR $\left(<60 \mathrm{~mL} / \mathrm{min} / 1.73 \mathrm{~m}^{2}\right)$, suggesting that both of these CKD markers are independent factors for dementia development [13]. The higher serum levels of cystatin C itself (corresponding to lower eGFR) were related to cognitive impairment measured by the Mini-Mental State Examination in another study conducted in Japan [29]. Together, these results suggest that apart from decreased eGFR, other factors related to higher cystatin $\mathrm{C}$ levels such as diabetes, higher levels of 

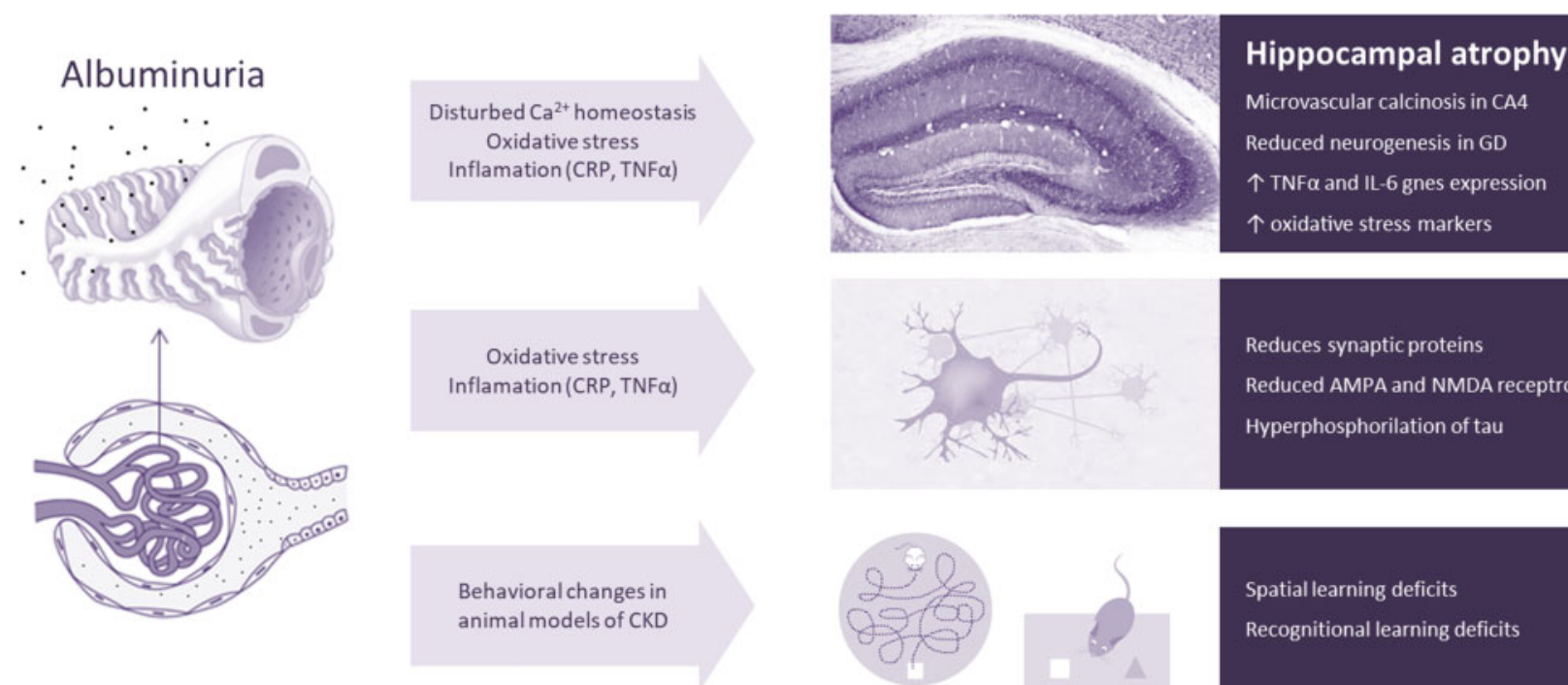

$\uparrow$ oxidative stress markers

Reduces synaptic proteins

Reduced AMPA and NMDA receptros

Hyperphosphorilation of tau

FIGURE 1: Hippocampal structural and functional changes in animal models of kidney disease. GD, gyrus dentatus.

C-reactive protein (CRP) and white blood cell count [30] could play a role in development of $\mathrm{MCI}$ or dementia that may further complicate the evaluation of its relationship to albuminuria.

\section{PATHOPHYSIOLOGY OF COGNITIVE IMPAIRMENT AND DEMENTIA IN ALBUMINURIC PATIENTS}

The multiple mechanisms explaining MCI and dementia in kidney dysfunction include vascular lesion and impaired cerebral blood flow autoregulation, neuroinflammation, effect of uraemic neurotoxins and kidney neurotrophins, and have been described in detail elsewhere [31,32]. Below we highlight the less studied pathways that link albuminuria and cognitive impairment. Albuminuria and an eGFR decrease could contribute to a reduction of hippocampal volume with concomitant microvascular damage, resulting in significant hippocampal anatomical and functional decline (Figure 1). The hippocampus is part of the limbic system of the brain and, among other physiological roles, is involved in the processes of formation, consolidation and memory retrieval [33]. The hippocampus is composed of several subregions including 'cornu ammonis' parts 1-4 (CA1-CA4), the 'dentate gyrus' and the 'subiculum'. $\mathrm{CA}$ regions contain three neuronal layers with pyramidal excitatory cells and are connected by several neural circuits with other parts of the hippocampus. The dentate gyrus is considered to be one of only two regions in the brain capable of adult neurogenesis and is thought to be involved in the maintenance of cognitive function, in particular spatial and non-spatial memory. However, different factors could be affecting it and a deficient rate of adult neurogenesis could potentially contribute to many neurological and psychiatric conditions.

Brain atrophy has been associated with physiological aging; however, in patients with $\mathrm{CKD}$, a higher prevalence of brain atrophy has been found, even at relatively young patients with mean age $60 \pm 12$ years [34]. In CKD patients, decline in GM volume seems to be more rapid and followed by cognitive impairment [35]. Studies in vivo performed using 3T-MRI scans showed that patients with CKD had smaller cerebral GM and hippocampal volume accompanied by decreased cortical thickness [36]. Also, disturbed calcium metabolism in CKD is associated with numerous neuropathological findings, including arteriosclerosis, microaneurysms and microvascular calcinosis, and the hippocampus is not spared from these pathological changes. Brain autopsy from subjects with CKD has demonstrated microvascular calcinosis in the CA4 hippocampal region [37]. These neuroanatomical changes correlated with poorer cognitive performance.

Furthermore, in an animal model of CKD, a reduction in synaptic proteins in the hippocampus was demonstrated at a molecular level. In unilateral ureteric obstruction (UUO) mice, a model of CKD, synapsin-1, synaptophysin and synaptotagmin, together with glutamate NMDAR2B and AMPA receptors, were all reduced compared with controls [38]. Even though the effect of albumin on neurocognitive parameters decay cannot be directly linked to CKD in this particular model, the study clearly demonstrates not only behavioural cognitive decline, but also reduced functional markers of excitatory hippocampal synapses [38], which should be investigated further in albuminuria models. Also, histological examination in UUO mice showed hyperphosphorylation of tau protein in the hippocampus.

Several lines of evidence show that functional performance in memory tests in animal models of CKD is associated with hippocampal decay and atrophy. Compared with the sham-operated group, in a mouse with CKD induced by UUO, weak performance was demonstrated in several behavioural learning and memory tests: novel object recognition test, Y-maze test and puzzle box test [38]. Spatial learning deficits were also demonstrated in the five-sixths nephrectomy $(5 / 6 \mathrm{Nx}) \mathrm{CKD}$ mouse model that resulted in increased escape latency during acquisition trials in the Moris water maze task and correlated with increased neuro-inflammatory markers in hippocampal tissue 
[39]. Unilateral nephrectomy (UniNx) and two-thirds electrocoagulation of the other renal cortex of C57BL/6 mice was associated with lower performance in the radial arm water maze test, and the UniNx mice made significantly more errors compared with sham-operated controls [40]. Cognitive impairment was accompanied by increased numbers of pyknotic neuronal cells in the hippocampus of the CKD mice.

Neuroanatomical and functional changes in the hippocampus of CKD patients and in animal models of CKD could be associated with reduced hippocampal neurogenesis. In patients with MCI, a lower number of neuroblast cells was found in the dentate gyrus of the hippocampus, while a higher expression level of these cells correlated with better cognitive scores [41]. Several factors could contribute to this, including chronic oxidative stress and increased glucocorticoid levels that reduce differentiation and proliferation of hippocampal neuronal stem cells, probably as a result of accumulation of mitochondrial oxidative stress [42, 43]. Accumulation of a critical marker of oxidative stress, 8-hydroxy-2'-deoxyguanosine, in hippocampal neuronal cells was found in an animal model of CKD [40]. Endoplasmic reticulum (ER) stress is also thought to contribute significantly to neuronal dysfunction and its role in the hippocampal dysfunction seen in CKD has been examined. The expression level of glucose-regulated protein 78 , a typical ER stress marker, showed a pronounced increase in the hippocampus. Furthermore, 4-hydroxy-2-nonenal-protein adducts, a marker of oxidative stress, was also increased in the hippocampus 8 weeks after $5 / 6 \mathrm{Nx}$ [44].

In UUO mice, increased levels of the inflammatory markers CRP and tumour necrosis factor- $\alpha$ (TNF- $\alpha$ ) in serum were detected, accompanied by elevated levels of the Nrf2 transcription factor and 8-hydroxyguanosine in the hippocampus [38]. Oxidant/antioxidant imbalance and up-regulation of TNF- $\alpha$ and interleukin-1 $\beta$ (IL-1 $\beta$ ) gene expression in hippocampal tissue were also detected in $5 / 6 \mathrm{Nx}$ mice. These changes were accompanied by increased proteinuria and a reduction in creatinine clearance [39]. Also, microglial activation should be considered as a source of neurotoxicity and release of proinflammatory factors that could contribute to cognitive impairment. Exposure of primary cultured microglia to serum albumin resulted in release of TNF- $\alpha$, transforming growth factor$\beta 1$ and higher inducible nitric oxide synthase expression [45].

Finally, blood-brain barrier (BBB) breakdown is an early event in the aging human brain that begins in the hippocampus and may contribute to cognitive impairment [46]. The BBB breakdown in the hippocampus and its CA1 and dentate gyrus subdivisions worsened with $\mathrm{MCI}$, and correlated with injury to BBB-associated pericytes seen in a mouse model of CKD [47]. Furthermore, it is known that small vessels in the kidney and brain are exposed to high blood flow volumes during the cardiac cycle [48]. Therefore, it has been hypothesized that kidney disease (as reflected in increased albuminuria) and cerebral small vessel disease (cognitive impairment) are both signs of systemic small vessel disease affecting different organs with anatomical and haemodynamic similarities. Moreover, endothelial dysfunction, regardless of the cause, leads to leakage of proteins into the interstitial space in both kidney and brain tissues [49].
Various mediators have been suggested to cause endothelial dysfunction. Particularly, kidney dysfunction induces nitric oxide deficiency due to disturbances in L-arginine metabolism, eventually affecting the maintenance of the microcirculation and BBB [49]. A key mechanism that may link albuminuria and BBB damage is a loss of the glycocalyx, a polysaccharide layer that lines the luminal endothelial surface and that acts as a barrier [50]. Degradation of the glycocalyx in response to endothelial activation, as may occur in kidney disease, can lead to albuminuria and increased microvascular permeability in organs other than the kidney, including the brain [50]. The cross-talk between kidney and brain may also involve the renin-angiotensin system [51], and the evidence from in vivo and clinical studies showing that the treatment with angiotensin-converting enzyme inhibitors and AT1 receptor blockers, beside exerting renoprotection, also has beneficial actions in neurodegenerative disorders [52].

\section{CONTROVERSIES IN THE RELATIONSHIP BETWEEN ALBUMINURIA AND MCI AND DEMENTIA}

Several controversies exist in the relationships between increased albuminuria and cognitive impairment, leaving the questions about its causal role. First, these two conditions share some important risk factors. Compared with the general population [53] or even other CKD patients with normal urinalysis $[6,54]$, patients with albuminuria are generally older and have more comorbidities, including higher prevalence of diabetes and cardiovascular disease, higher smoking rate and higher $\mathrm{BP}-$ each of these can affect brain function over time. In fact, the Cardiovascular Health Cognition Study demonstrated the role of such confounders in patients who had doubling of albuminuria over time: after adjustment for cardiovascular disease and demographic factors the relative risk of dementia development was substantially attenuated, and the risk of mild cognitive decline becomes non-significant [6]. Diabetes alone increases the odds of cognitive decline by 1.2- to 1.7 -fold, depending on the examination tool used $[55,56]$. Even young patients with type 1 diabetes, who had good glycaemic control and few comorbidities, over 18 years of follow-up demonstrated a decline in psychomotor and motor speed (but not in memory or intelligence tests). In this cohort several major predictors of cognitive dysfunction have been revealed, including a rise in serum creatinine or the need for dialysis, but the role of albuminuria has not been studied [57].

Second, albuminuria may be the result of systemic endothelial dysfunction that also plays an independent role in $\mathrm{MCI}$ and dementia development $[58,59]$. Notably, both the kidney and brain have low vascular resistance systems and can maintain stable continuous high-volume perfusion that is resistant to fluctuations in systemic BP, although this feature makes both organs sensitive to disturbances in endothelial function and autoregulation, and stiffness of central arteries [48]. The design of studies performed so far is hampered by the inability to evaluate endothelial dysfunction and its role on MCI or dementia, since most studies analyse 'CKD in general' without any fine-grain distinction by the primary cause of CKD or types of 
macro- and microvascular abnormalities that may be present. The data on cognitive decline in different diseases that affect kidney structure are lacking, and only analyses with a mixture of primary (glomerular diseases) and secondary (diabetes, hypertensive nephropathy, etc.) kidney aetiologies are currently available. The dilemma of whether kidney dysfunction in primary nephropathies leads to systemic endothelial dysfunction that subsequently increases the risk of MCI and dementia, or whether the primary systemic endothelial dysfunction per se is due to diseases affecting multiple organs and leads to the lesion in both kidney and brain, remains unresolved. Moreover, there are only a few, and not always unidirectional [6], published data on whether an increase or decrease of albuminuria over time has any impact on progression of $\mathrm{MCI}$ and dementia.

Third, incident cognitive dysfunction can be limited by intensive BP control as demonstrated in the systolic blood pressure intervention trial memory and cognition in decreased hypertension (SPRINT MIND) study [60] in which the intensively treated group ( $\mathrm{SBP}<120 \mathrm{mmHg}$ ) had a substantially lower hazard ratio (HR) at 0.81 [95\% confidence interval (CI) 0.69-0.95] for development of MCI compared with the standard treatment group (SBP $<140 \mathrm{mmHg}$ ). However, this effect should be studied further in relation to overt kidney disease, because in subgroup analysis the patients with CKD had a HR of 1.00 (95\% CI 0.77-1.31) for this endpoint (even if no distinction between low eGFR and albuminuria in patients has been carried out), suggesting that the onset of cognitive impairment can be reduced by intensive BP control only in patients without CKD. The relationship between BP and albuminuria is complex, and a secondary analysis of the SPRINT trial [54] has shown that in the intensively treated group the association of albuminuria and stroke was not significant [1.25 (95\% CI 0.692.28)], while in the standard treatment group this association was present [3.44 (95\% CI 2.11-5.61)]. The effect of risk factors correction that may improve cognitive status in the general population [61, 62] - namely, additional physical activity, cognitive training, a Mediterranean diet, obesity correction, reduction of social isolation and smoking cessation-remain unknown in CKD patients, and the benefits of their correction have never been studied considering the relationship between albuminuria and MCI or dementia.

Finally, MCI is a rather broad term, covering different cognitive domains [31], including memory, reaction time, attention, executive function, concentration, visuospatial performance and others. Dementia is also non-heterogeneous condition that has distinct nosologic forms, including Alzheimer's disease, vascular dementia, Lewy body dementia and frontotemporal dementia. The widely used Montreal Cognitive Assessment (MoCA) covers different domains of cognitive abilities, but it may have different results for some domains compared with other tests. For example, only $7 \%$ of patients had abnormal trail making test (TMT), which examines executive function (visual attention and task switching), while $44 \%$ had abnormal MoCA, and proteinuria was substantially higher in those with abnormal TMT (median protein-to-creatinine ratio 150 versus $43 \mathrm{~g} / \mathrm{mol}$, respectively) [63]. Only some of the studies discussed here have applied a comprehensive evaluation of all cognitive domains
Table 1. Recommendations for further clinical studies on relationship between albuminuria and MCI or dementia

1. Collect data about comorbidities and risk factors (DM, hypertension, smoking, etc.) that could lead to development of MCI or dementia.

2. Account for the primary kidney disease that has led to albuminuria development.

3. Account for markers of systemic inflammation (CRP) at baseline and during follow-up.

4. Evaluate the effect of change in albuminuria, eGFR, blood pressure, glucose level, body mass index on MCI or dementia during follow-up.

5. Evaluate in CKD patients the effect of non-pharmacological interventions (additional physical activity, cognitive training, etc.) that could lead to prevention of MCI and dementia.

6. Apply in the same study several tests/questionnaires that evaluate different cognitive domains and include tests that allow to cross-validate impairment in a single cognitive domain.

7. Use imaging techniques to identify instrumental signs of brain dysfunction in CKD patients.

8. Estimate indicators of endothelial autoregulation disturbances (measuring flow-mediated dilatation), stiffness of central arteries (measuring pulse wave velocity) and retinal vessels analysis to evaluate the role of vascular system in development of MCI or dementia in CKD patients.

9. Evaluate joint effect of albuminuria and eGFR on MCI or dementia in prospective studies. Use equations for calculation of eGFR based on both serum creatinine and cystatin C.

10. In case of dementia as outcome, report the exact nosological form (Alzheimer's disease, vascular dementia, Lewy body dementia and frontotemporal dementia) and provide study findings for each of them.

with several tests in the same patients that allow to study the role of albuminuria and other factors in the complex landscape of MCI and dementia domains. All these conundrums will need to be addressed in future studies (Table 1).

\section{CONCLUSIONS}

We have summarized evidence that delineates some of the possible pathophysiological mechanisms and clinical significance of albuminuria for MCI and dementia. Despite the availability of strong evidence for an association between albuminuria and MCI or dementia, several important knowledge gaps exist, leaving unresolved the major questions about a causal relationship and the effectiveness of interventions targeting albuminuria per se to prevent cognitive decline. Our recommendations for future research aim to help in planning clinical trials and to solve this complex conundrum, with the ultimate goal to improve the outcomes in patients with kidney damage.

\section{ACKNOWLEDGEMENTS}

This publication is based upon work from the Cognitive Decline in Nephro-Neurology: European Cooperative Target (CONNECT) Action CA19127, supported by European Cooperation in Science and Technology (COST), www.cost. eu. The extended information about the action is available at www.connectcost.eu.

\section{FUNDING}

This article is published as part of a supplement financially supported by the COST Action CA19127-Cognitive 
Decline in Nephro-Neurology: European Cooperative Target (CONNECT).

\section{CONFLICT OF INTEREST STATEMENT}

B.B., G.H., V.P., A.F., F.M.-R., G.C., A.B. and N.P. declare no conflict of interest. M.J.S. reports personal fees from NovoNordisk, Jansen, Boehringer, Mundipharma, AstraZeneca, Esteve, Fresenius, Ingelheim Lilly, Vifor, ICU medical and Bayer during the conduct of the study. R.U. is currently employed by AstraZeneca BioPharmaceuticals R\&D, Early Cardiovascular, Renal and Metabolism, Cambridge UK and Gothenburg Sweden. M.E. reports grants from Bayer and fees paid to the Charité from AstraZeneca, Bayer, Boehringer Ingelheim, BMS, Daiichi Sankyo, Amgen, GSK, Sanofi, Covidien, Novartis and Pfizer, all outside the submitted work. G.R. reports consulting fees from Akebia Pharmaceuticals Inc., Alexion Pharmaceuticals Inc., BioCryst Pharmacauticals Inc. and Janssen Research \& Development LLC, speaker honorarium and travel reimbursement from Alnylam, Boehringer Ingelheim and Incaption Sciences Canada.

\section{APPENDIX}

\section{CONNECT collaborators are}

Giovambattista Capasso; Alexandre Andrade; Maie Bachmann; Inga Bumblyte; Adrian Constantin Covic; Pilar Delgado; Nicole Endlich; Andreas Engvig; Denis Fouque; Casper Franssen; Sebastian Frische; Liliana Garneata; Loreto Gesualdo; Konstantinos Giannakou; Dimitrios Goumenos; Ayşe Tuğba Kartal; Laila-Yasmin Mani; Hans-Peter Marti; Christopher Mayer; Rikke Nielsen; Vesna Pšić; Merita Rroji (Molla); Giorgos Sakkas; Goce Spasovski; Kate I. Stevens; Evgueniy Vazelov; Davide Viggiano; Lefteris Zacharia; Ana Carina Ferreira; Jolanta Malyszko; Ewout Hoorn; Andreja Figurek; Robert Unwin; Carsten A. Wagner; Christoph Wanner; Annette Bruchfeld; Marion Pepin; Andrzej Wieçek; Dorothea Nitsch; Ivo Fridolin; Gaye Hafez; Maria José Soler; Michelangela Barbieri; Bojan Batinić; Laura Carrasco; Sol Carriazo; Ron Gansevoort; Gianvito Martino; Francesco Mattace Raso; Ionut Nistor; Alberto Ortiz; Giuseppe Paolisso; Daiva Rastenyte; Gabriel Stefan; Gioacchino Tedeschi; Ziad A. Massy; Boris Bikbov; Karl Hans Endlich; Olivier Godefroy; Jean-Marc Chillon; Anastassia Kossioni; Justina Kurganaite; Norberto Perico; Giuseppe Remuzzi; Tomasz Grodzicki; Francesco Trepiccione; Carmine Zoccali; Mustafa Arici; Peter Blankestijn; Kai-Uwe Eckardt; Danilo Fliser; Eugenio Gutiérrez Jiménez; Maximilian König; Ivan Rychlik; Michela Deleidi; George Reusz.

\section{REFERENCES}

1. KDIGO 2012 clinical practice guideline for the evaluation and management of chronic kidney disease. Kidney Int Suppl 2013; 3: 1-150

2. Piccoli GB, Cabiddu G, Attini R et al. Risk of adverse pregnancy outcomes in women with CKD. J Am Soc Nephrol 2015; 26: 2011-2022

3. Bikbov B, Purcell CA, Levey AS et al. GBD Chronic Kidney Disease Collaboration. Global, regional, and national burden of chronic kidney disease, 1990-2017: a systematic analysis for the Global Burden of Disease Study 2017. Lancet 2020; 395: 709-733

4. Vos T, Lim SS, Abbafati C et al. Global burden of 369 diseases and injuries in 204 countries and territories, 1990-2019: a systematic analysis for the Global Burden of Disease Study 2019. Lancet 2020; 396: 1204-1222

5. Abbatecola AM, Barbieri M, Rizzo MR et al. Arterial stiffness and cognition in elderly persons with impaired glucose tolerance and microalbuminuria. J Gerontol Ser A Biol Sci Med Sci 2008; 63: 991-996

6. Barzilay JI, Fitzpatrick AL, Luchsinger J et al. Albuminuria and dementia in the elderly: a community study. Am J Kidney Dis 2008; 52: 216-226

7. Joosten H, Izaks GJ, Slaets JPJ et al. Association of cognitive function with albuminuria and eGFR in the general population. Clin J Am Soc Nephrol 2011; 6: 1400-1409

8. Martinez-Vea A, Salvadó E, Bardají A et al. Silent cerebral white matter lesions and their relationship with vascular risk factors in middle-aged predialysis patients with CKD. Am J Kidney Dis 2006; 47: 241-250

9. Weiner DE, Bartolomei K, Scott T et al. Albuminuria, cognitive functioning, and white matter hyperintensities in homebound elders. Am J Kidney Dis 2009; 53: 438-447

10. Akoudad S, Sedaghat S, Hofman A et al. Kidney function and cerebral small vessel disease in the general population. Int J Stroke 2015; 10: 603-608

11. Yamasaki K, Hata J, Furuta Y et al. Association of albuminuria with white matter hyperintensities volume on brain magnetic resonance imaging in elderly Japanese - the Hisayama study. Circ J 2020; 84: 935-942

12. Sedaghat S, Ding J, Eiriksdottir G et al. The AGES-Reykjavik Study suggests that change in kidney measures is associated with subclinical brain pathology in older community-dwelling persons. Kidney Int 2018; 94: 608-615

13. Scheppach JB, Coresh J, Wu A et al. Albuminuria and estimated GFR as risk factors for dementia in midlife and older age: findings from the ARIC study. Am J Kidney Dis 2020; 76: 775-783

14. Takae K, Hata J, Ohara $\mathrm{T}$ et al. Albuminuria increases the risks for both Alzheimer disease and vascular dementia in community-dwelling Japanese elderly: the Hisayama study. J Am Heart Assoc 2018; 7: e006693

15. Barzilay JI, Lovato JF, Murray AM et al. Albuminuria and cognitive decline in people with diabetes and normal renal function. Clin J Am Soc Nephrol 2013; 8: 1907-1914

16. Mehta D, Pimentel DA, Núñez MZ et al. Subclinical albuminuria is linked to gray matter atrophy in type 2 diabetes mellitus. Metabolism 2014; 63: 1390-1397

17. Barzilay JI, Morgan TM, Murray AM et al. Brain MRI volume findings in diabetic adults with albuminuria: the ACCORD-MIND study. Gerona 2016 71: 803-810

18. Freedman BI, Sink KM, Hugenschmidt CE et al. Associations of early kidney disease with brain magnetic resonance imaging and cognitive function in African Americans with type 2 diabetes mellitus. Am J Kidney Dis 2017; 70: 627-637

19. Barzilay JI, Younes N, Pop-Busui R et al. The cross-sectional association of renal dysfunction with tests of cognition in middle-aged adults with early type 2 diabetes: the GRADE study. J. Diabetes Complications 2021; 35: 107805

20. Moulignier A, Viret-Vilayphon AC, Lescure FX et al:; ALCOVE Study Group. Microalbuminuria: a sentinel of neurocognitive impairment in HIV-infected individuals? J Neurol 2020; 267: 1368-1376

21. Rüth EM, Landolt MA, Neuhaus TJ et al. Health-related quality of life and psychosocial adjustment in steroid-sensitive nephrotic syndrome. J Pediatr 2004; 145: 778-783

22. Guha P, De A, Ghosal M. Behavior profile of children with nephrotic syndrome. Indian J Psychiatry 2009; 51: 122-126

23. Manti P, Giannakopoulos G, Giouroukou E et al. Psychosocial and cognitive function in children with nephrotic syndrome: association with disease and treatment variables. Biopsychosoc Med 2013; 7: 10

24. Bakkum L, Willemen AM, Zoetebier L et al. A longitudinal study on the effects of psychological stress on proteinuria in childhood steroid-sensitive nephrotic syndrome. J Psychosom Res 2019; 121: 8-13

25. Huang JA, Lin $\mathrm{CH}$, Chang YT et al. Nephrotic syndrome is associated with increased risk of ischemic stroke. J Stroke Cerebrovasc Dis 2019; 28: 104322

26. Berger I, Wu S, Masson P et al. Cognition in chronic kidney disease: a systematic review and meta-analysis. BMC Med 2016; 14: 206 
27. Tseng TJ, Yen YT, Yang YH et al. Association between the occurrence of albuminuria and the risk of early dementia among older people upon health examination: a community-based cohort study in Taiwan. BMJ Open 2020; 10: e041664

28. Gabin JM, Romundstad S, Saltvedt I et al. Moderately increased albuminuria, chronic kidney disease and incident dementia: the HUNT study. BMC Nephrol 2019; 20: 261

29. Kono S, Adachi H, Enomoto $\mathrm{M}$ et al. Impact of cystatin $\mathrm{C}$ and microalbuminuria on cognitive impairment in the population of community-dwelling Japanese. Atherosclerosis 2017; 265: 71-77

30. Stevens LA, Schmid CH, Greene T et al. Factors other than glomerular filtration rate affect serum cystatin C levels. Kidney Int 2009; 75: 652-660

31. Viggiano D, Wagner CA, Martino G et al. Mechanisms of cognitive dysfunction in CKD. Nat Rev Nephrol 2020; 16: 452-469

32. Drew DA, Weiner DE, Sarnak MJ. Cognitive impairment in CKD: pathophysiology, management, and prevention. Am J Kidney Dis 2019; 74: 782-790

33. Wong EYH, Herbert J. Raised circulating corticosterone inhibits neuronal differentiation of progenitor cells in the adult hippocampus. Neuroscience 2006; 137: 83-92

34. Tsuruya K, Yoshida H, Kuroki Y et al. Brain atrophy in peritoneal dialysis and CKD stages 3-5: a cross-sectional and longitudinal study. Am J Kidney Dis 2015; 65: 312-321

35. Tsuruya K, Yoshida H. Brain atrophy and cognitive impairment in chronic kidney disease. Contribut Nephrol 2018; 196: 27-36

36. Chang CY, Lin CC, Tsai CF et al. Cognitive impairment and hippocampal atrophy in chronic kidney disease. Acta Neurol Scand 2017; 136: 477-485

37. Vinters HV, Magaki SD, Williams CK. Neuropathologic findings in chronic kidney disease (CKD). J Stroke Cerebrovasc Dis 2021; 30: 105657

38. Ho YS, Lau CF, Lee K et al. Impact of unilateral ureteral obstruction on cognition and neurodegeneration. Brain Res Bull 2021; 169: 112-127

39. Askari H, Abazari MF, Ghoraeian P et al. Ameliorative effects of hydrogen sulfide (NaHS) on chronic kidney disease-induced brain dysfunction in rats: implication on role of nitric oxide (NO) signaling. Metab Brain Dis 2018; 33: 1945-1954

40. Fujisaki K, Tsuruya K, Yamato $\mathrm{M}$ et al. Cerebral oxidative stress induces spatial working memory dysfunction in uremic mice: neuroprotective effect of tempol. Nephrol Dial Transplant 2014; 29: 529-538

41. Tobin MK, Musaraca K, Disouky A et al. Human hippocampal neurogenesis persists in aged adults and Alzheimer's disease patients. Cell Stem Cell 2019; 24: 974-982.e3

42. Wang Y, Neumann M, Hansen K et al. Fluoxetine increases hippocampal neurogenesis and induces epigenetic factors but does not improve functional recovery after traumatic brain injury. J Neurotrauma 2011; 28: 259-268

43. Chetty S, Friedman AR, Taravosh-Lahn K et al. Stress and glucocorticoids promote oligodendrogenesis in the adult hippocampus. Mol Psychiatry 2014; 19: 1275-1283

44. Kosuge Y, Osada N, Shimomura A et al. Relevance of the hippocampal endoplasmic reticulum stress response in a mouse model of chronic kidney disease. Neurosci Lett 2018; 677: 26-31

45. Hooper C, Pinteaux-Jones F, Fry VAH et al. Differential effects of albumin on microglia and macrophages; implications for neurodegeneration following blood-brain barrier damage. J Neurochem 2009; 109: 694-705
46. Montagne A, Barnes SR, Sweeney MD et al. Blood-brain barrier breakdown in the aging human hippocampus. Neuron 2015; 85: 296-302

47. Nakagawa T, Hasegawa Y, Uekawa K et al. Chronic kidney disease accelerates cognitive impairment in a mouse model of Alzheimer's disease, through angiotensin II. Exp Gerontol 2017; 87: 108-112

48. O'Rourke MF, Safar ME. Relationship between aortic stiffening and microvascular disease in brain and kidney. Hypertension 2005; 46: 200-204

49. Knopman DS, Mosley TH, Bailey KR et al. Associations of microalbuminuria with brain atrophy and white matter hyperintensities in hypertensive sibships. J Neurol Sci 2008; 271: 53-60

50. Rabelink TJ, de Zeeuw D. The glycocalyx-linking albuminuria with renal and cardiovascular disease. Nat Rev Nephrol 2015; 11: 667-676

51. Miranda AS, Cordeiro TM, Dos Santos Lacerda Soares TM et al. Kidneybrain axis inflammatory cross-talk: from bench to bedside. Clin Sci (Lond) 2017; 131: 1093-1105

52. Villapol S, Saavedra JM. Neuroprotective effects of angiotensin receptor blockers. Am J Hypertens 2015; 28: 289-299

53. Jonsson AJ, Lund SH, Eriksen BO et al. The prevalence of chronic kidney disease in Iceland according to KDIGO criteria and age-adapted estimated glomerular filtration rate thresholds. Kidney Int 2020; 98: 1286-1295

54. Leitão L, Soares-Dos-Reis R, Neves JS et al. Intensive blood pressure treatment reduced stroke risk in patients with albuminuria in the SPRINT trial. Stroke 2019; 50: 3639-3642

55. Cukierman T, Gerstein HC, Williamson JD. Cognitive decline and dementia in diabetes - Systematic overview of prospective observational studies. Diabetologia 2005; 48: 2460-2469

56. Mccrimmon RJ, Ryan CM, Frier BM. Diabetes 2 diabetes and cognitive dysfunction. Lancet 2012; 379: 2291-2299

57. Jacobson AM, Ryan CM, Cleary PA et al.; Diabetes Control and Complications Trial/EDIC Research Group. Biomedical risk factors for decreased cognitive functioning in type 1 diabetes: an 18 year follow-up of the Diabetes Control and Complications Trial (DCCT) cohort. Diabetologia 2011; 54: 245-255

58. Deckert T, Feldt-Rasmussen B, Borch-Johnsen K et al. Albuminuria reflects widespread vascular damage. The Steno hypothesis. Diabetologia 1989; 32: 219-226

59. Ruggenenti P, Remuzzi G. Time to abandon microalbuminuria? Kidney Int 2006; 70: 1214-1222

60. Williamson JD, Pajewski NM, Auchus AP. Effect of intensive vs. standard blood pressure control on probable dementia: a randomized clinical trial. JAMA 2019; 321: 553-561

61. Livingston $\mathrm{G}$, Sommerlad A, Orgeta V et al. Dementia prevention, intervention, and care. Lancet 2017; 390: 2673-2734

62. Valls-Pedret C, Sala-Vila A, Serra-Mir M et al. Mediterranean diet and agerelated cognitive decline. JAMA Intern Med 2015; 175: 1094-1103

63. Tollitt J, Odudu A, Montaldi D et al. Cognitive impairment in patients with moderate to severe chronic kidney disease: the Salford kidney cohort study. Clin Kidney J 2021; 14: 1639-1648

Received: 30.5.2021; Editorial decision: 16.8.2021 